\title{
L'éducation thérapeutique, au cœur de la prise en charge du patient obèse
}

\author{
J.-P. Bastard · J. Dargent $\cdot$ M. Pigeyre \\ (C) Lavoisier SAS 2014
}

Chers lecteurs d'Obésité, comme depuis plusieurs années maintenant, nous vous présentons dans ce numéro de décembre 2014, les résumés des communications qui seront présentées au prochain congrès de l'AFERO qui se tiendra à Nantes les 15 et 16 janvier prochain.

Vous trouverez aussi dans ce numéro un rapport très complet sur l'éducation thérapeutique et le parcours de soins de la personne obèse réalisé par un groupe d'experts dans le domaine et piloté par $\mathrm{O}$. Ziegler. Fera écho à ce référentiel un article d'A. Grimaldi sur l'éducation thérapeutique. Une belle mise au point sur l'obésité et la stéatose hépatique nous est proposée par R. Anty et al. Un sujet tout à fait original sur les effets cellulaires induits par les fibres alimentaires nous est présenté par F. De Valder et G. Mithieux. Tout aussi original est l'approche de S. Hafiz et al dans l'utilisation des technologies innovantes (ultrasons diffus associés au travail musculaire sur plaque vibrante) pour tenter de modifier la composition corporelle de sujets modérément obèses. Dans un article original, $\mathrm{M}$. Baguma et al nous exposent les problèmes posés par l'utilisation des critères classiques du syndrome métabolique chez les patientes multipares en République Démocratique du Congo. Nous retrouverons aussi la revue de presse présentée par L. Genser et C. Barrat ainsi qu'un cas clinique de chirurgie didactique présenté par F. Barros et al.

Nous espérons que vous prendrez plaisir à lire ce numéro d'Obésité! 